\title{
Synthesis of 2,4,5-Trisubstituted Pyrimidines from Baylis-Hillman Adducts and Amidines
}

\author{
Jeong Mi Kim, Sung Hwan Kim, and Jae Nyoung Kim* \\ Department of Chemistry and Institute of Basic Science, Chonmam National University, Gwangiu 500-757, Korea \\ "E-mail: kimin@chonnamackr. \\ Received Angust 20, 2007
}

Key Words : Pyrimidines, Baylis-IIilman adducts, $\Lambda$ midines

The pyrimidine moiety is one of the most widespread heterocycles in biologically occurring compounds, such as nucleic acids and vitamin $\mathrm{B}_{1}$, and is an important constituent of numerous drug molecules in many therapeutic areas. ${ }^{1-4}$ Although various procedures for the synthesis of pyrimidine derivatives have been developed, ${ }^{2-4}$ it is convenient to synthesize substituted pyrimidines by the reaction of amidine or guanidine derivatives with a variety of 1,3-dielectrophilic three-carbon units such as $\alpha, \beta$-unsaturated carbonyl compounds. ${ }^{3.4}$

During the continuous studies on the chemical transformation of Baylis-Hillman adducts our recent interest was focused on the synthesis of oxygen and nitrogen-containing heterocyclic compounds. ${ }^{+6}$ In addition, we were interested in the reaction of Baylis-Hillman adducts and 1,3-dinucleophile like as 1,3-dinitroalkanes ${ }^{6}$ and dimethy] 1,3acetonedicarboxylate. ${ }^{6}$ In these respects we presumed that we could synthesize trisubstituted pyrimidine derivatives 3 by using some amidine derivatives 2 as shown in Scheme 1 . In the reaction, Baylis-Hillman acetates $\mathbf{1}$ act as 1,3dielectrophilic component and amidine derivatives $\mathbf{2}$ served the role of 1,3-dinucleophile.

The reaction of the Baylis-Hillman acetate 1a and benzamidine hydrochloride ( $2 \mathbf{a}$ ) in tert-butanol in the presence of $\mathrm{K}_{2} \mathrm{CO}_{3}$ produced desired compound $3 \mathbf{a}$ in $91 \%$ isolated yield (entry I in Table I). Similarly we prepared 2,4,5-trisubstituted pyrimidines $\mathbf{3 b} \mathbf{b}$ in $27-92 \%$ yjelds from the reaction of Baylis-Hillman acetates 1a-f and amidine derivatives 2a and $\mathbf{2 b}$, and the results are summarized in Table 1. As shown in Table 1 , the reaction of acetamidine hydrochloride (2b)
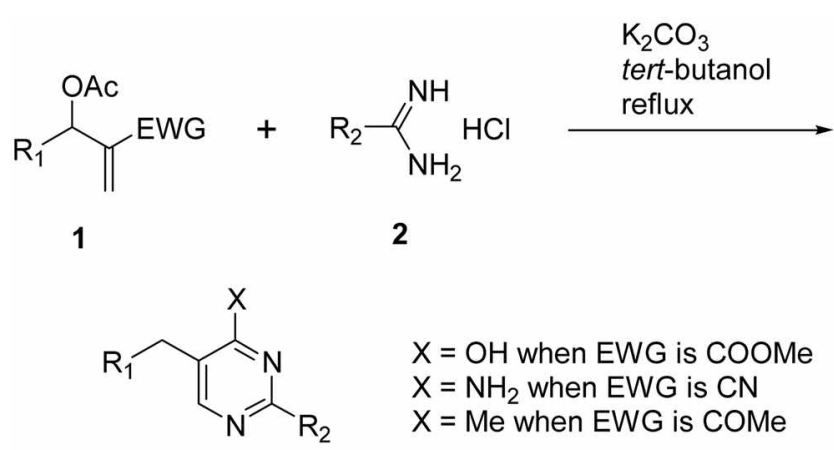

3

Scheme 1 gave relatively lower yields than the cases of $\mathbf{2 a}$ (see entries $1 \& 2$, entries $6 \& 7$ ) presumably due to low solubility of $2 \mathrm{~b}$ in tert-butanol. The Baylis-Hillman acetates containing ester moiety (entries 1-5) or acetyl group (entries 6 and 7) showed moderate to good reactivity, however, nitrile-containing substrate $1 \mathrm{f}$ showed very low reactivity (entry 8 ). The reaction of 1a and 1,3-diphenyl guanidine (2c) gave the corresponding 2-aminopyrimidine derivative $\mathbf{3 i}$ in moderate yield (entry 9 ). ${ }^{4 b}$ Initially we examined the reaction of BaylisHillman adduct itself and benzamidine hydrochloride (2a), however, we could not obtain the desired product 3a directly.

In summary, we synthesized some 2,4,5-trisubstituted pyrimidines from the reaction of Baylis-Hillman acetates and amidine derivatives in a one-pot reaction in moderate yields.

\section{Experimental Section}

Typical procedure for the synthesis of 3a. A stirred solution of $1 \mathrm{a}(234 \mathrm{mg}, 1.0 \mathrm{mmol}), 2 \mathrm{a}(235 \mathrm{mg}, 1.5 \mathrm{mmol})$, and $\mathrm{K}_{2} \mathrm{CO}_{3}(276 \mathrm{mg}, 2.0 \mathrm{mmol})$ in tert-butanol $(3 \mathrm{~mL})$ was heated to reflux for $3 \mathrm{~h}$. After usual aqueous extractive workup and column chromatographic purification process $\left(\mathrm{CH}_{2} \mathrm{Cl} / \mathrm{EtOAc}, 5: 2\right)$ we obtained 3a $(239 \mathrm{mg}, 91 \%)$ as a white solid. The spectroscopic data of 3a-i are as follows.

Compound 3a: $91 \%$; white solid, $\mathrm{mp} 235-237^{\circ} \mathrm{C}$ (dec.); IR (film) $3068,2950,1645 \mathrm{~cm}^{-1}$; ${ }^{3} \mathrm{H}$ NMR (CDCl 3,300 $\mathrm{MHz}) \delta 3.86(\mathrm{~s}, 2 \mathrm{H}), 7.18-7.35(\mathrm{~m}, 5 \mathrm{H}), 7.46-7.55(\mathrm{~m}, 3 \mathrm{H})$, $7.99(\mathrm{~s}, 1 \mathrm{H}), 8.19-8.22(\mathrm{~m}, 2 \mathrm{H}), 13.18(\mathrm{br} \mathrm{s}, 1 \mathrm{H}) ;{ }^{13} \mathrm{C} \mathrm{NMR}$ $\left(\mathrm{CDCl}_{3}, 75 \mathrm{MHz}\right) \delta 33.47,126.02,126.46,127.43,128.50$, $128.92(2 \mathrm{C}), 131.79,132.01,138.71,152.98,155.67,164.40$.

Compound 3b: $75 \%$; white solid, mp $175-177^{\circ} \mathrm{C}$; IR (film) $3345,2927,1714,1667 \mathrm{~cm}^{-1} ;{ }^{1} \mathrm{H}$ NMR $\left(\mathrm{CDCl}_{3}, 300\right.$ MHz) $\delta 2.40(\mathrm{~s}, 3 \mathrm{H}), 3.76(\mathrm{~s}, 2 \mathrm{H}), 7.17-7.34(\mathrm{~m}, 5 \mathrm{H}), 7.76$ (s, $1 \mathrm{H}), 13.30$ (br s, $1 \mathrm{H}) ;{ }^{13} \mathrm{C} \mathrm{NMR}\left(\mathrm{CDCl}_{3}, 75 \mathrm{MHz}\right) \delta$ $21.24,33.13,124.99,126.37,128.39,128.89,138.67$, $152.95,157.49,164.91$.

Compound 3e: $92 \%$; white solid, mp $236-238{ }^{\circ} \mathrm{C}$ (dec.); IR (film) $2920,2850,1651,1574 \mathrm{~cm}^{-1}$; $\mathrm{H}$ NMR (CDCl, $300 \mathrm{MHz}) \delta 2.30(\mathrm{~s}, 3 \mathrm{H}), 3.81(\mathrm{~s}, 2 \mathrm{H}), 7.08(\mathrm{~d}, J=7.8 \mathrm{~Hz}$, $2 \mathrm{H}), 7.21(\mathrm{~d}, J=7.8 \mathrm{~Hz}, 2 \mathrm{H}), 7.47-7.58(\mathrm{~m}, 3 \mathrm{H}), 7.98(\mathrm{~s}$, $1 \mathrm{H}), 8.19-8.22(\mathrm{~m}, 2 \mathrm{H}), 13.12(\mathrm{br} \mathrm{s}, 1 \mathrm{H}) ;{ }^{13} \mathrm{C} \mathrm{NMR}\left(\mathrm{CDCl}_{3}\right.$, $75 \mathrm{MHz}) \delta 21.01,33.07,126.18,127.53,128.80,128.86$, 
Table 1. Synthesis of 2,4,5-trisubstituted pyrimidines

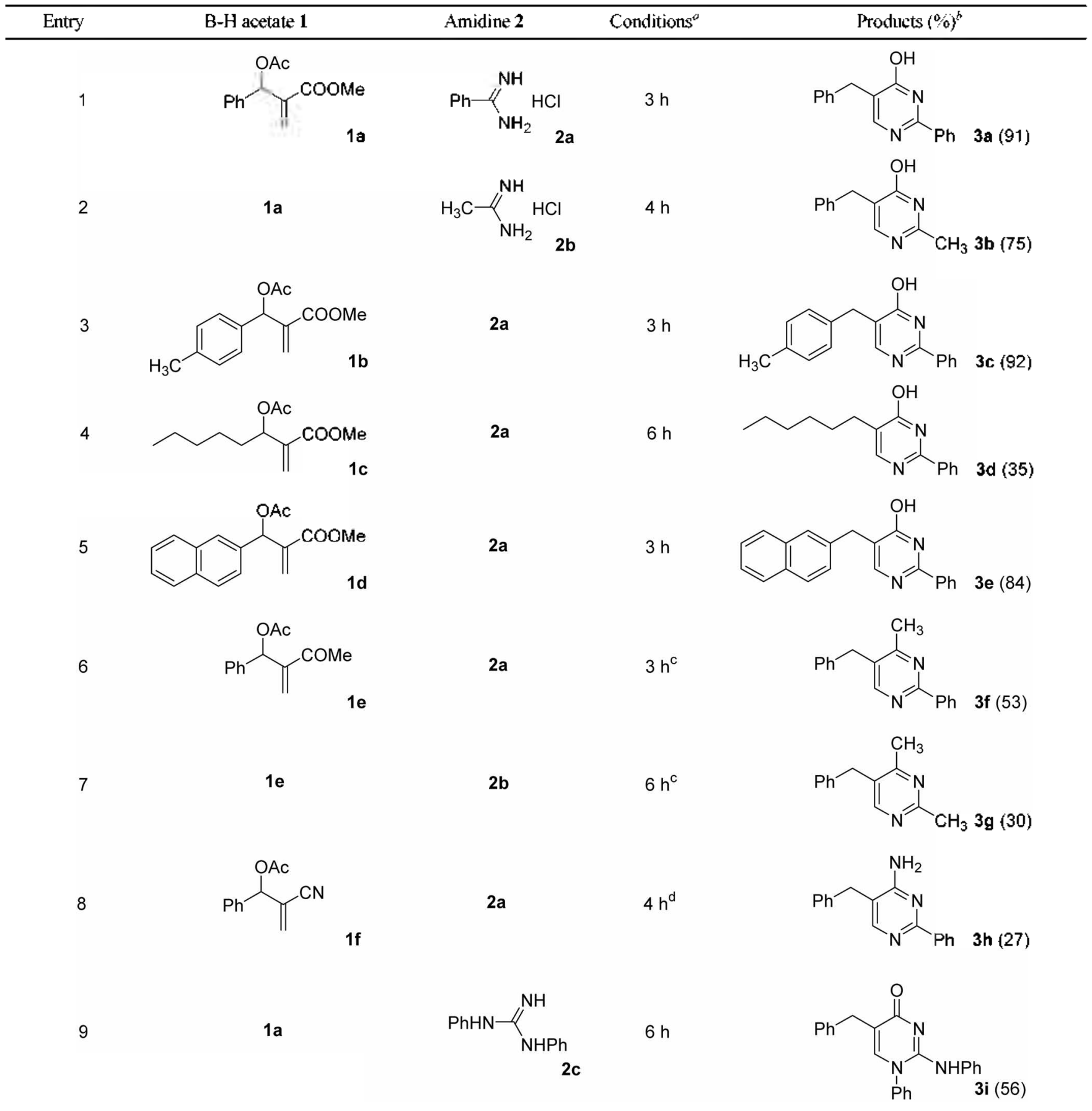

"Conditions: $\mathrm{B}-\mathrm{H}$ acelate ( 1.0 equiv), amidine ( 1.5 equiv), $\mathrm{K}_{3} \mathrm{CO}_{3}$ (2.0 equiv), tert-butanol, rellux. 'Tsolated yield. Conditions: $\mathrm{B}-\mathrm{H}$ acelate ( 1.5 equiv), amidine (1.0 equiv), $\mathrm{K}_{2} \mathrm{CO}_{3}\left(2.0\right.$ equiv), tert-butanol, reflux. ${ }^{d} \mathrm{Conditions:} \mathrm{B-II} \mathrm{acetate} \mathrm{(} 1.0$ equiv), amidine ( 3.5 equiv), $\mathrm{K}_{2} \mathrm{CO}_{3}(4.0$ equiv), tert-butanol, reflux.

$129.16,131.71,132.07,135.66,135.97,152.90,155.67,164.60$. Compound 3d: $35 \%$; white solid, $\mathrm{mp} 122-124{ }^{\circ} \mathrm{C}$; IR (film) 3406, 3070, 2927, 2852, $1643 \mathrm{~cm}^{-1}$; ${ }^{1} \mathrm{H} \mathrm{NMR}$ (CDCl ${ }_{3}$, $300 \mathrm{MHz}) \delta 0.90(\mathrm{t}, J=6.9 \mathrm{~Hz}, 3 \mathrm{H}), 1.26-1.45(\mathrm{~m}, 6 \mathrm{H})$, $1.61-1.71(\mathrm{~m}, 2 \mathrm{H}), 2.54(\mathrm{t}, J=7.2 \mathrm{~Hz}, 2 \mathrm{H}), 7.48-7.58(\mathrm{~m}$, $3 \mathrm{H}), 7.98(\mathrm{~s}, \mathrm{IH}), 8.20-8.25(\mathrm{~m}, 2 \mathrm{H}), 12.86$ (br s, $1 \mathrm{H}) ;{ }^{13} \mathrm{C}$ NMR $\left(\mathrm{CDCl}_{3}, 75 \mathrm{MHz}\right) \delta 14.09,22.65,27.58,28.22,29.17$, $31.65,127.03,127.34,128.88,131.64,132.14,152.23$, $155.16,164.74$.
Compound 3e: 84\%; white solid, mp $269-271{ }^{\circ} \mathrm{C}$ (dec.); IR (film) $3377,2917,2850,1643 \mathrm{~cm}^{-1}$; 'H NMR (CDCl $300 \mathrm{MHz}) \delta 4.02(\mathrm{~s}, 2 \mathrm{H}), 7.41-7.55(\mathrm{~m}, 6 \mathrm{H}), 7.72-7.81(\mathrm{~m}$ $4 \mathrm{H}), 7.98(\mathrm{~s}, 1 \mathrm{H}), 8.06-8.08(\mathrm{~m}, 2 \mathrm{H}), 11.57(\mathrm{br} \mathrm{s}, \mathrm{IH}) ;{ }^{13} \mathrm{C}$ NMR (DMSO-d $6,75 \mathrm{MHz}) \delta 32.77,125.38,126.03,126.60$, $127.36,127.45,127.46,127.50,127.55,127.56,127.76,128.61$, $131.40,131.69,133.09,137.15,151.53,155.94,163.04$.

Compound 3f: $53 \%$; white solid, mp $68-69^{\circ} \mathrm{C}$; IR (film) $3030,2923,1574,1541,1426 \mathrm{~cm}^{-1}$; $H$ NMR (CDCl 3,300 
MHz) $\delta 2.49(\mathrm{~s}, 3 \mathrm{H}), 3.99(\mathrm{~s}, 2 \mathrm{H}), 7.12-7.15(\mathrm{~m}, 2 \mathrm{H}), 7.20-$ $7.33(\mathrm{~m}, 3 \mathrm{H}), 7.44-7.51(\mathrm{~m}, 3 \mathrm{H}), 8.41-8.46(\mathrm{~m}, 2 \mathrm{H}), 8.47(\mathrm{~s}$, $1 \mathrm{H}) ;{ }^{13} \mathrm{C} \mathrm{NMR}\left(\mathrm{CDCl}_{3}, 75 \mathrm{MHz}\right) \delta 22.40,35.87,126.66$, $127.93,128.49,128.53,128.75,129.45,130.27,137.73$, $138.15,157.22,162.73,166.13$.

Compound 3g: 30\%; colorless oil; IR (film) 3042, 2926, $1581,1556,1440 \mathrm{~cm}^{-1}$; 'H NMR (CDCl $\left.3,300 \mathrm{MHz}\right) \delta 2.39$ $(\mathrm{s}, 3 \mathrm{H}), 2.68(\mathrm{~s}, 3 \mathrm{H}), 3.94(\mathrm{~s}, 2 \mathrm{H}), 7.08-7.1 \mathrm{l}(\mathrm{m}, 2 \mathrm{H}), 7.20-$ $7.33(\mathrm{~m}, 3 \mathrm{H}), 8.32(\mathrm{~s}, 1 \mathrm{H}){ }^{13} \mathrm{C} \mathrm{NMR}\left(\mathrm{CDCl}_{3}, 75 \mathrm{MHz}\right) \delta$ $22.09,25.62,35.68,126.64,128.42,128.45,128.73,138.15$, $157.02,165.86,165.93$.

Compound 3h: $27 \%$; white solid, mp $176-178{ }^{\circ} \mathrm{C}$; IR (film) $3481,3286,3066,1641 \mathrm{~cm}^{-1}$; $\mathrm{H}$ NMR (CDCl, 300 MHz) $\delta 3.85(\mathrm{~s}, 2 \mathrm{H}), 4.79$ (br s, 2H), 7.19-7.24 (m, 2H), 7.25-7.36 (m, 3H), 7.41-7.46 (m, 3H), $8.24(\mathrm{~s}, 1 \mathrm{H}), 8.31-$ $8.35(\mathrm{~m}, 2 \mathrm{H}) ;{ }^{13} \mathrm{C} \mathrm{NMR}\left(\mathrm{CDCl}_{3}, 75 \mathrm{MHz}\right) \delta 34.78,113.71$, $127.10,127.81,128.28,128.33,129.03,130.08,137.09$, $137.93,156.18,161.83,163.31$.

Compound 3i: $56 \%$; yellow solid, mp $160-162{ }^{\circ} \mathrm{C}$; IR (film) $3431,2254,2127,1647 \mathrm{~cm}^{-1}$; $\mathrm{H}$ NMR (CDCl, 300 MHz) $\delta 4.80(\mathrm{~d}, J=1.8 \mathrm{~Hz}, 2 \mathrm{H}), 6.86(\mathrm{br} \mathrm{s}, 2 \mathrm{H}), 7.01-7.06$ (m, lH), 7.26-7.31 (m, 5H), 7.37-7.44 (m, 7H), 7.57 (br s, $1 \mathrm{H}), 7.87(\mathrm{~s}, 1 \mathrm{H})$. We took the ${ }^{13} \mathrm{C}$ NMR spectrum of compound $\mathbf{3 i}$, however, we could not assign the peaks definitely due to line broadening. Thus we prepared $N$-allyl derivative ( $3 \mathrm{i}$, allyl bromide, $\mathrm{K}_{2} \mathrm{CO}_{3}, \mathrm{DMF}, \mathrm{rt}, 2 \mathrm{~h}, 66 \%$ ) and confirmed the structure of $\mathbf{3 i}$, and the spectroscopic data of $\mathrm{N}$-allyl derivative of $3 \mathrm{i}$ are as follows: $66 \%$; pale yellow oil; IR (film) $1627,1589,1493,1417,1377 \mathrm{~cm}^{-1}$; 'H NMR $\left(\mathrm{CDCl}_{3}\right.$, $300 \mathrm{MHz}) \delta 4.61(\mathrm{~d}, J=1.5 \mathrm{~Hz}, 2 \mathrm{H}), 4.84(\mathrm{~d}, J=5.4 \mathrm{~Hz}$, $2 \mathrm{H}), 5.26(\mathrm{dd}, J=10.2$ and $1.5 \mathrm{~Hz}, 1 \mathrm{H}), 5.38(\mathrm{dd}, J=17.1$ and $1.5 \mathrm{~Hz}, 1 \mathrm{H}), 6.06-6.20(\mathrm{~m}, 1 \mathrm{H}), 6.53-6.56(\mathrm{~m}, 2 \mathrm{H}), 6.61-$ $6.66(\mathrm{~m}, 1 \mathrm{H}), 6.72-6.81(\mathrm{~m}, 3 \mathrm{H}), 6.86-7.00(\mathrm{~m}, 4 \mathrm{H}), 7.12-7.15$ $(\mathrm{m}, 2 \mathrm{H}), 7.30-7.32(\mathrm{~m}, 3 \mathrm{H}), 7.81(\mathrm{~s}, \mathrm{IH}) ;{ }^{13} \mathrm{C} \mathrm{NMR}\left(\mathrm{CDCl}_{3}\right.$, $75 \mathrm{MHz}) \delta 44.83,49.50,116.87,121.58,121.79,124.32$, $124.39,125.29,127.95,128.60,128.66,129.10,129.41$, $133.48,134.27,137.51,144.01,144.63,147.51,164.77$.

Acknowledgements. This work was supported by the program of KOSEF (R\&E of Science High School, 200701-049) and the authors wish to thank Kyoung Do Jung, Dae Han Kim, Kyung June $\mathrm{Oh}$, Sang Ha Lee for their involvement in this project. Spectroscopic data was obtained from the Korea Basic Science Institute, Gwangju branch.

\section{References and Notes}

1. (a) Brown, D. J. The Primidines; John Wiley \& Sons: New York, 1962; pp 31-81. (b) Holfmann, M. G; Nowak, A.; Muller, M. Pyrimidines in Methods of Organic Chemistry (Houben-Weyl); Schaumann, E., Ed.; Georg Thieme Verlag: Stuttgart, 1998; Vol. E9b/Part I. Hetarenes IV, pp 1-249. (c) Brown, D. J. Conprehensive Heterocyclic Chemistiy II, Katritzky, A. R., Rees, C. W., Eds.; Pergamon Press: Oxford, 1996; Vol, 3, Chapter 2.13.

2. For recent examples on the synthesis of pyrimidines, see: (a) Muller, T. J. J.; Braun, R.; Ansorge, M. Org. Lett. 2000, 2, 19671970. (b) Sakai, N.; Aoki, Y.; Sasada, T.; Konakahara, T. Org. Lett. 2005, 7, 4705-4708. (c) Yoon, D. S.; Han, Y.; Stark, T. M.;
Haber, J. C.; Gregg, B. T.; Stankovich, S. B. Org. Lett. 2004, 6, 4775-4778. (d) Movassaghi, M.; Hill, M. D. J. Am. Chem. Soc. 2006, 128, 14254-14255. (e) Kakiya, H.; Yagi, K.; Shinokubo, H.; Oshima, K. J. An. Chem. Soc. 2002, 124, 9032-9033. (f) Cesar, J. J. Comb. Chem. 2005, 7, 517-519. (g) Xie, F.; Li, S.; Bai, D.; Lou, L.; Hu, Y. J. Comb. Chem. 2007, 9, 12-13. (h) Herrera, A.; Martinez-Alvarez, R.; Chioua, M.; Chioua, R.; Sanchez, A. Tetrahedron 2002, 58, 10053-10058. (i) Kawamura, S.; Sanemitsu, Y. J. Org. Chem. 1993, 58, 414.418.

3. For the synthesis of pyrimidines by the reaction of 1,3-dielectrophilic components and amidines or guanidines, see: (a) Bellur, E; Langer, P. Tetrahedron 2006, 62, 5426-5434. (b) Karpov, A. S.; Merkul, E.; Rominger, F.; Muller, T. J. J. Angew. Chem, Int. Ed. 2005, 44, 6951-6956. (c) Funabiki, K.; Nakamura. H.; Matsui, M.; Shibata, K. Sintett 1999, 756-758. (d) Hawksley, D.; Griffin, D. A.; Leeper, F. J. J. Chem. Soc., Perkin Trans. I 2001, 144-148. (e) Pirc, S.; Bevk, D.; Golobic, A.; Stanovnik, B.; Svete, J. Helv. Chim. Acta 2006, 89, 30-44.

4. For the synthesis of pyrimidine derivatives from Baylis-Hillman adducts, see: (a) Lee, C. G.; Gowrisankar, S.; Kim, J. N. Bull. Korean Chen. Soc. 2005, 26, 481-484. (b) Basavaiah, D.; Satyanarayana, T. Tetrahedron Lett. 2002, 43, 4301-4303. (c) Pathak, R.; Roy, A. K.; Batra, S. Syylett 2005, 848-850.

5. Recent reports on the synthesis of oxygen and nitrogen-containing compounds from Baylis-Hillman adducts, see: (a) Lee, K. Y.; Lee, H. S.; Kim, J. N. Tetrahedron Lett. 2007, 48, 2007-20 I1. (b) Kim, S. J.; Lee. H. S.; Kim. J. N. Tetrahedron Lett. 2007. 48, $1069-$ 1072. (c) Gowrisankar, S.; Kim, S. J.; Kim, J. N. Tetrahedron Lett. 2007, 48, 289-292. (d) Gowrisarkar, S.; Lee, K. Y.; Kim, T. H.; $\mathrm{Kim}, \mathrm{J}$. N. Tetrahedron Lett, 2006, 47,5785-5788. (e) Kim, S. C. Lee, H. S.; Lee, Y. J.; Kim, J. N. Tetrahedron Lett. 2006, 47, 56815685. (f) Lee, K. Y.; Lee. H. S.; Kim, J. N. Bull. Korean Chen. Soc. 2007, 28, 333-335. (g) Lee, C. G; Lee, K. Y.; Kim, S. J.; Kim. J. N. Bull. Korean Chem. Soc. 2007. 28, 719-720.

6. Recent publications on the reaction of 1,3-dinucleophiles and the Baylis-Hillman adducts, see: (a) Park, D. Y.; Lee, K. Y.; Kim, J. N. Tetrahedron Lett, 2007, 48, 1633-1636. (b) Park, D. Y,; Kim, S. J.; Kim, T. H.; Kim, J. N. Tetrahedron Lett. 2006, 47,6315-6319.

7. The reaction of Baylis-Hillman adduct itself and $2 \mathbf{a}$ is summarized in Scheme 2. The reaction in tert-butanol in the presence of $\mathrm{K}_{2} \mathrm{CO}_{3}$ showed the formation of diastereomeric mixture of 4 $(58 \%, \mathrm{~L}: 1)$, dihydropyrimidine $5(15 \%)$, and benzaldehyde 6 $(13 \%)$. Dihydropyrimidine 5 and benzaldehyde 6 might be produced by retro-Baylis-Hillman process from 4 . When we carried out the reaction in ethanol we observed rapid decomposition of initially formed 4 into intractable polar compounds. The compound 4 could be transformed into product $3 a$ by dehydration ( $p$ - $\mathrm{TsOH}$, coluene, reflux, I h) and the following isomerization $\left(\mathrm{K}_{2} \mathrm{CO}_{3}\right.$, tert-butanol, reflux, $1 \mathrm{~h}$ ) in $35 \%$ yield.

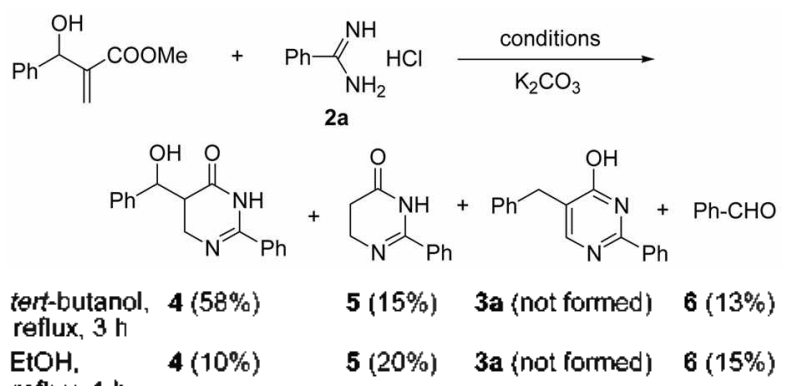

reflux, $1 \mathrm{~h}$

Scheme 2

8. (a) Weis, A. L.; Zamir, D. J. Org. Chem. 1987, 52, 3421-3425. (b) Einsiedel, J.; Hubner, H.; Gmeiner, P. Bioorg. Med. Chem. Lett. 2003, I3, 85I-854. 